\title{
ISO 6789 under revision - proposals for calibration results of hand torque tools including measurement uncertainty
}

\author{
Dirk Röske \\ Physikalisch-Technische Bundesanstalt, Bundesallee 100, 38116 Braunschweig, Germany
}

\section{ABSTRACT}

The ISO 6789:2003 standard is widely used as a technical document for the calibrations of hand torque tools, but it does not require any statements about uncertainties of the calibration results. Nevertheless, according to the GUM it is necessary to report a complete measurement result including its uncertainty. In addition, according to ISO/IEC 17025:2005, calibration laboratories must take into account measurement uncertainties in the case that they make a conformity declaration in their calibration certificates. In this paper, a proposal is given on how to estimate measurement uncertainties of ISO 6789 calibration results taking into account various influencing quantities.

\section{Section: RESEARCH PAPER \\ Keywords: hand torque tools; torque wrenches; calibration; ISO 6789:2003; uncertainty}

Citation: Dirk Röske, ISO 6789 under revision - proposals for calibration results of hand torque tools including measurement uncertainty, Acta IMEKO, vol. 3, no. 2, article 7, June 2014, identifier: IMEKO-ACTA-03 (2014)-02-07

Editor: Paolo Carbone, University of Perugia

Received February $20^{\text {th }}, 2013$; In final form December $7^{\text {th }}, 2013$; Published June 2014

Copyright: @ 2014 IMEKO. This is an open-access article distributed under the terms of the Creative Commons Attribution 3.0 License, which permits unrestricted use, distribution, and reproduction in any medium, provided the original author and source are credited

Corresponding author: Dirk Röske, e-mail: dirk.roeske@ptb.de

\section{INTRODUCTION}

The ISO 6789:2003 standard [1] is widely used as a guide for the calibration of torque wrenches and other hand-operated torque tools like screwdrivers indicating the applied torque, or setting torque tools. Unfortunately, this standard does not contain a method or even a proposal for the uncertainty calculation of the measurement results obtained in these calibrations. On the other hand, according to the GUM [2] it is necessary to report a complete measurement result including its uncertainty. This problem was the subject of an earlier work which was based on the previous issue of the standard [3] and did not take into account some important influences such as the connecting profile (often the square drive) and the point of force application (lever length, cross forces and bending moments). This paper describes a proposal on how the standard could be improved in order to become a complete calibration guide. Methods for calculating calibration results and estimating their uncertainties will be presented. The paper will point out influencing quantities which have not been taken into account until now and will deal with some specialties of torque tool calibration devices. The subject matter of this calibration guide is also the focus of discussions within the Deutscher Kalibrierdienst (DKD) - the recently (re-)founded German board for technical cooperation between PTB and torque calibration laboratories. Some conclusions resulting from these discussions are also mentioned in this paper.

\section{GENERAL ISO PROCEDURE}

The ISO 6789:2003 standard deals with two types of hand torque tools: Type I - indicating torque tools and Type II setting torque tools (see Figure 1). For both of these types various classes are defined, such as torque wrenches or screwdrivers utilizing a scale, a gauge or an electronic display. Some of these devices have a housing, some have a fixed measuring point, others are adjustable.

In general, the calibration procedure consists of one (Type I) respectively five (Type II) pre-loadings with the maximum torque, without recording the values and a subsequent series of five (for two classes of Type II: ten) repeated applications of three different torques from the measurement range. The three torque values are defined by the standard as $20 \%$, approximately $60 \%$ (depending on the value that can be adjusted on the scale) and $100 \%$ of the maximum (nominal)

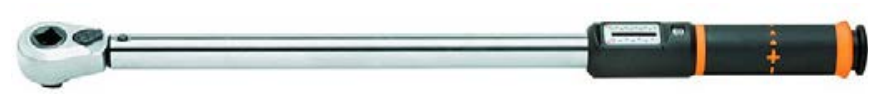

Figure 1. Setting torque wrench model 3360, [4]. 
torque of the tool.

Special conditions for the loading have to be fulfilled for setting torque tools: an increasing force rising to approximately $80 \%$ of the target value has to be applied first. Then the force has to be constantly and slowly increased up to $100 \%$ of the target value within a time window of $0.5 \mathrm{~s} \ldots 4 \mathrm{~s}$. A calibration facility for setting torque tools must take this requirement into account.

\section{CALIBRATION FACILITIES}

There are some other important remarks to be made with respect to the calibration facilities. First of all, they are very diverse. We know, for example, facilities that utilize lever-mass systems and a supported lever with air bearing for generating a pure torque. The torque tool or torque wrench is connected to the measuring axis of the facility and fixed or supported on the opposite side near the handle. These facilities offer the smallest uncertainties.

The majority of the calibration facilities use integrated torque transducers and they are calibrated with torque transfer wrenches. The torque is generated then by either rotating the torque transducer against the fixed or supported tool or by applying a force at the handle of the torque wrench perpendicular to the beam and the torque axis. These systems allow the torque application to be controlled with the help of the torque transducers used as references. This is often the more accurate and reliable procedure, but it cannot be applied to setting torque tools. In these cases the torque must be increased until the setting torque is reached. If a calibration facility and the measurement results obtained are to be considered, it must be clear whether the calibration procedure was controlled by the facility's calibration torque or by the tool to be calibrated.

Another topic is related to the signals recorded by the data acquisition. Most of the torque transducers used in the facilities are strain-gauge based and deliver nearly exclusively signals in $\mathrm{mV} / \mathrm{V}$ or $\mathrm{N} \cdot \mathrm{m}$. These can be set to zero (be tared) before starting the measurements or not. In addition, when the calibration procedure for measuring tools says that a certain torque has to be applied, then it is not always the best idea to adjust this value as well as possible. Especially in such cases when the force is applied with a manually operated crank on a spindle, this can take a long time, even for a well-trained person. If the torque is as close to the target value as necessary, then the value can be recorded. Sometimes it may be useful to calculate the result for the calibration torque using a regression function.

\section{CALIBRATION RESULTS ACCORDING TO THE EXISTING STANDARD ISO 6789:2003}

According to the current version of the standard (published in 2003), the relative deviation $A_{\mathrm{s}}$ in \% must be calculated from $\mathrm{A}_{\mathrm{s}}(\%)=\frac{\mathrm{X}_{\mathrm{a}}-\mathrm{X}_{\mathrm{r}}}{\mathrm{X}_{\mathrm{r}}} \cdot 100$.

Here $X_{\mathrm{a}}$ is the torque indicated by the tool and $X_{\mathrm{r}}$ is the reference torque, i.e. the calibration torque measured by the calibration facility. In the special case of Type II, classes $C$ and $\mathrm{F}$ tools (without scale), the value of $X_{\mathrm{a}}$ is calculated as the mean of the ten reference torques $X_{\mathrm{r}}$ measured by the calibration facility. This value is then used in equation (1).
As stated above, no uncertainty of the input or resulting values is given. There is just one requirement regarding the calibration facility: the maximum uncertainty of the indicated torque must be $\pm 1 \%$.

The conformity can be declared if the deviations are within the limit set by the standard ( $\pm 4 \%$, or $\pm 6 \%$ for some classes). Again, there is no measurement uncertainty taken into account in contradiction to the IEC/ISO 17025:2005 standard ("General requirements for the competence of testing and calibration laboratories") which requires conformity statements to be made taking the uncertainty of measurement into account [5]. This is very important for accredited calibration laboratories.

\section{PROPOSED IMPROVEMENTS}

There are a number of possible improvements to the ISO 6789:2003 standard. All of them have been discussed within the torque committee of the DKD.

Calibration range: Within the $\mathrm{DKD}$ committee it was proposed to choose the starting point of the tool's measurement range as the first calibration point instead of $20 \%$ of the full scale. This would be more useful from the point of view of the tool's application and is especially important for indicating torque tools which are used in a wide measuring range.

Calibration facility's operation mode: Calibration facilities usually utilize torque transducers or sensors of precision torque wrenches as sensing elements. These sensors are calibrated as single devices or as part of the whole facility. It is quite interesting that the ISO 6789 standard does not offer the possibility that the torque is controlled by the calibration facility's sensor but the tool under calibration has to be loaded with increasing force (at the end of the beam) until it indicates the corresponding torque. On the other hand, automated calibration procedures can be realized much more easily if the torque is controlled by the facility's sensor and not by the tool. In addition, if the tool shows completely invalid values, then wrong torques can be applied. It is proposed to allow the torque application to be controlled by the calibration facility's indication. This is hereinafter referred to as case A. In the case that the torque application is controlled by the tool, we propose calling this case B. Of course, this discussion does not completely apply to Type II torque tools since their reaction (click) is essential for the calibration.

Designations: Calibration certificates are sometimes not very clear with respect to the numbers shown in their tables. It would be very helpful to offer some unique designations for the quantities to be measured. Taking into account different possible options, it is important to clearly distinguish between

- the indications recorded during the calibration: indications of the calibration facility and those of the tool, both can be gross or net (tared) values,

- the calibration facility's operation mode: case A or case B (see above), and

- the calibration procedure, i.e. if the calibration torques are tuned in (by hand or electronic control) or if a subsequent interpolation is necessary.

The designations given in Tables 1 and 2 are proposed.

Calibration result: For the user of a measuring instrument it is sometimes of interest not only to know if the tool is within the specifications (conformity), but also to know the value of the deviation and the uncertainty of this value. Especially with 
indicating tools, the deviation found during the calibration can be compensated when the tools are used in an application. This is, of course, an additional effort, but the reward is a more

Table 1. Proposed designations for the indications of the calibration facility and the tool to be calibrated.

\begin{tabular}{cl}
\hline Designation & \multicolumn{1}{c}{ Description } \\
\hline$i$ & $\begin{array}{l}\text { Ordinal number of the torque step (for example } \\
i=1: 20 \mathrm{~N} \cdot \mathrm{m}, i=2: 60 \mathrm{~N} \cdot \mathrm{m}, i=3: 100 \mathrm{~N} \cdot \mathrm{m}, i=4:-20 \mathrm{~N} \cdot \mathrm{m} \\
\text { and so on) }\end{array}$ \\
\hline$j$ & $\begin{array}{l}\text { Ordinal number of the single value measured for torque } \\
\text { step } i(j=1,2, \ldots 5, \text { or } j=1,2, \ldots \text { 10) }\end{array}$ \\
\hline$J_{0, i, j}$ & $\begin{array}{l}\text { Zero signal indicated by the calibration facility prior to } \\
\text { the measurement of value } j \text { for torque step } i\end{array}$ \\
\hline$J_{\mathrm{M}, i, j}$ & $\begin{array}{l}\text { Measurement signal indicated by the calibration facility } \\
\text { during the measurement of value } j \text { for torque step } i\end{array}$ \\
\hline$M_{\mathrm{K}, i, j}$ & $\begin{array}{l}\text { Tared calibration torque in the measurement of value } j \\
\text { for torque step } i\end{array}$ \\
\hline$I_{0, i, j}$ & $\begin{array}{l}\text { Zero signal indicated by the tool under calibration prior } \\
\text { to the measurement of value } j \text { for torque step } i\end{array}$ \\
\hline$I_{\mathrm{M}}$ & $\begin{array}{l}\text { Measurement signal indicated by the tool under } \\
\text { calibration during the measurement of value } j \text { for torque } \\
\text { step } i\end{array}$ \\
\hline$Y_{\mathrm{M}}$ & $\begin{array}{l}\text { Tared measuring value in the measurement of value } j \text { for } \\
\text { torque step } i\end{array}$ \\
\hline
\end{tabular}

accurate and reliable measurement. It is now proposed to calculate an additional calibration result for each torque step (and each direction of the torque, if necessary) as the mean of the five or ten single values found for this torque step during the calibration.

According to the two operation modes, the calibration result is a value calculated from the indications of the tool (case A)

$Y_{i}=Y\left(M_{\mathrm{N}, i}\right)=\frac{1}{n} \sum_{j=1}^{n} Y_{\mathrm{A}, i, j}$,

or from the indications of the calibration facility (case B)

$M_{i}=\frac{1}{n} \sum_{j=1}^{n} M_{\mathrm{A}, i, j}$.

It is important to understand that in both cases the

Table 2. Proposed designations taking into account the facility's operation mode and, if necessary, interpolation.

\begin{tabular}{cl}
\hline Designation & \multicolumn{1}{c}{ Description } \\
\hline Case A & $\begin{array}{l}\text { Nominal calibration torque for torque step } \\
i \text { (all } j)\end{array}$ \\
\hline$M_{\mathrm{N}, i}$ & $\begin{array}{l}\text { The tool's tared and interpolated measuring value for the } \\
\text { measurement of the single value } j \text { for torque step } i\end{array}$ \\
\hline$Y_{\mathrm{A}, i, j}$ & The tool's calibration result for torque step $i$ \\
\hline$Y_{i}$ & $\begin{array}{l}\text { Nominal calibration torque of the tool for torque step } i \\
\text { (all } j \text { ) }\end{array}$ \\
\hline$Y_{\mathrm{N}, i}$ & $\begin{array}{l}\text { Tared and interpolated calibration torque for the } \\
\text { measurement of the single value } j \text { for torque step } i\end{array}$ \\
\hline$M_{\mathrm{A}, i, j}$ & The facility's calibration result for torque step $i$ \\
\hline$M_{i}$ &
\end{tabular}

calibration torque is defined by the calibration facility. The deviations $f_{\mathrm{q}, i}$ are always calculated as differences between the torque value of the tool and that of the calibration facility, regardless of the facility's operation mode.
Influencing quantities: It is known that many quantities can influence the calibration result of a torque tool and most of them are not mentioned in the standard. This is normally not a problem when dealing with mechanical torque tools with permissible deviations of $4 \%$ or $6 \%$. But in the last few years electronic tools with claimed uncertainties of $1 \%$ have come onto the market. On the other hand, comparison measurements have shown that connecting parts, such as square drives, can have a significant influence, especially when the results are close to the permissible limits.

These effects are mainly caused by the geometrical dimensions, manufacturing tolerances and material properties (hardness, heat treatment) of the connecting elements (often square drives or other types of profiles). Sometimes these parts are worn out but there is no criterion saying that they must be replaced.

The influence of the connecting profile $b_{\mathrm{V}}$ can be measured using the following method: for a number of $k=1,2, \ldots m$ (for example, $m=4$ for square drives) equally distributed over $360^{\circ}$ positions of the profile, $l=1,2, \ldots o$ single measurements (for example, $o=10$ ) have to be carried out in each position, i.e. for $m=4$ and $o=10$, in total 40 measurements. The applied torque should be the starting point of the tool's measurement range with a nominal beam length defined by the middle of the handle.

The parameter $b_{\mathrm{V}}$ can then be calculated

- in case A from

$b_{\mathrm{V}}=\max _{k}\left(\frac{1}{o} \sum_{l=1}^{o} Y_{\mathrm{A}, k, l}\right)-\min _{k}\left(\frac{1}{o} \sum_{l=1}^{o} Y_{\mathrm{A}, k, l}\right)$,

and

- in case B from

$b_{\mathrm{V}}=\max _{k}\left(\frac{1}{o} \sum_{l=1}^{o} M_{\mathrm{A}, k, l}\right)-\min _{k}\left(\frac{1}{o} \sum_{l=1}^{o} M_{\mathrm{A}, k, l}\right)$.

Another possible influence is the position of the force introduction point of torque wrenches, i.e. the beam length. For constant torque, an increasing beam length corresponds to a decreasing force. This effect is not applicable to screwdrivers.

The influence of the beam length can be measured using the following method: at $60 \%$ of the nominal torque, $l=1,2, \ldots o$ single measurements (for example, $o=10$ ) have to be carried out with nominal beam length (defined by the middle of the handle), with the beam length increased by $10 \mathrm{~mm}$ and with the mean beam length decreased by $10 \mathrm{~mm}$, i.e. for $o=10$, in total 30 measurements.

The parameter $b_{\mathrm{L}}$ can then be calculated

- in case A from

$$
\begin{aligned}
b_{\mathrm{L}}= & \max \left(\left|\frac{1}{o} \sum_{l=1}^{o} Y_{\mathrm{A}, \text { mean }, l}-\frac{1}{o} \sum_{l=1}^{o} Y_{\mathrm{A}, \text { long }, l}\right|,\right. \\
& \left.\left|\frac{1}{o} \sum_{l=1}^{o} Y_{\mathrm{A}, \text { mean }, l}-\frac{1}{o} \sum_{l=1}^{o} Y_{\mathrm{A}, \text { short }, l}\right|\right), \text { and } \\
& \text { - in case B from } \\
b_{\mathrm{L}}= & \max \left(\left|\frac{1}{o} \sum_{l=1}^{o} M_{\mathrm{A}, \text { mean }, l}-\frac{1}{o} \sum_{l=1}^{o} M_{\mathrm{A}, \text { long }, l}\right|,\right. \\
& \left.\left|\frac{1}{o} \sum_{l=1}^{o} M_{\mathrm{A}, \text { mean }, l}-\frac{1}{o} \sum_{l=1}^{o} M_{\mathrm{A}, \text { short }, l}\right|\right) .
\end{aligned}
$$




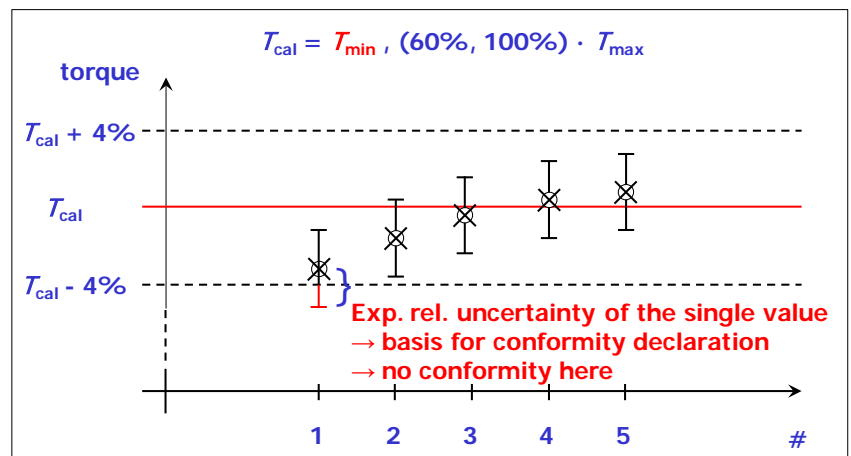

Figure 2. Conformity evaluation using uncertainty.

Here, "mean" means nominal beam length, "long" is nominal beam length increased by $10 \mathrm{~mm}$, and "short" is nominal beam length decreased by $10 \mathrm{~mm}$.

Many setting hand torque tools have a ratchet included. So the question arises of how the calibration results change if different positions of this ratchet are used. This effect is partly included in parameter $b_{\mathrm{V}}$ if the ratchet is used to change the position of the connecting profile. Otherwise an additional measurement with all possible positions of the ratchet should be considered, but this can be quite time-consuming. The author's working group has started to collect such values and would be very grateful for any assistance.

Environmental influences play an important role in many calibrations. This is partly taken into account by the standard's requirement that the temperature should be constant $\left( \pm 1^{\circ} \mathrm{C}\right)$ within an interval of $18^{\circ} \mathrm{C}$ to $28^{\circ} \mathrm{C}$. Ambient humidity must be recorded and be lower than or equal $90 \%$ (relative humidity). This value is too high. One problem is that steel parts begin to rust when exposed to very high humidity. Another problem may be the changing viscosity of lubricants inside mechanical tools thus affecting their mechanical properties. It is expected that changing temperatures have a similar effect.

How can all these influences be treated? Worn square drives or ratchets must be replaced. Extreme temperatures and humidities should be avoided. Thus, it is proposed to include at least the influence of the connecting parts' geometry $b_{\mathrm{V}}$ and the position of the force introduction point (beam length) $b_{\mathrm{L}}$. In order to keep the calibration effort at a justifiable level, it is not necessary to measure the corresponding parameters every time the tool is calibrated and for every single measuring instrument, however, typical values for the given type of tool should be used instead. We think it is sufficient to use an estimate for these parameters for a series of tools with the same design and make. The manufacturers are asked to provide these values. Only in the case of worn out connectors, suspected damage of the tool, or other evidence of deviations, the complete measurement is useful.

Uncertainties: It is proposed to calculate the parameter repeatability $b_{i}$ from the five (or ten) single measurement values for each torque step as a span maximum - minimum:

$$
\text { - in case A from }
$$$$
b_{i}=b\left(Y_{i}\right)=\max _{k}\left(Y_{\mathrm{A}, i, j}\right)-\min _{k}\left(Y_{\mathrm{A}, i, j}\right) \text {, and }
$$

- in case B from

$b_{i}=b\left(M_{i}\right)=\max _{k}\left(M_{\mathrm{A}, i, j}\right)-\min _{k}\left(M_{\mathrm{A}, i, j}\right)$.

Together with $b_{\mathrm{V}}$ and $b_{\mathrm{L}}$, another parameter must be taken into account: the resolution $r$ of the tool, as defined in the standard. The influence of the interpolation $f_{\mathrm{a}}$ can often be kept
Table 3. Proposed distributions and corresponding standard uncertainties for important parameters.

\begin{tabular}{|c|c|c|}
\hline \multirow{2}{*}{$\begin{array}{c}\text { Parameter, } \\
\text { evaluation type and } \\
\text { distribution } \\
\text { function }\end{array}$} & \multicolumn{2}{|c|}{$\begin{array}{l}\text { Relative standard uncertainty } w \\
\text { in \% }\end{array}$} \\
\hline & case $A$ & case $B$ \\
\hline $\begin{array}{c}r \\
\text { type B, } \\
\text { rectangular }\end{array}$ & $w_{\mathrm{r}}=\frac{\left(\frac{r}{2}\right)}{\sqrt{3}} \frac{100}{M_{\mathrm{N}, i}}$ & $w_{\mathrm{r}}=\frac{\left(\frac{r}{2}\right)}{\sqrt{3}} \frac{100}{Y_{\mathrm{N}, i}}$ \\
\hline $\begin{array}{c}b_{i} \\
\text { type B, } \\
\text { rectangular }\end{array}$ & $w_{b, i}=\frac{\left(\frac{b_{i}}{2}\right)}{\sqrt{3}} \frac{100}{M_{\mathrm{N}, i}}$ & $w_{b, i}=\frac{\left(\frac{b_{i}}{2}\right)}{\sqrt{3}} \frac{100}{Y_{\mathrm{N}, i}}$ \\
\hline $\begin{array}{c}b_{\mathrm{V}} \\
\text { type } \mathrm{B}, \\
\text { rectangular }\end{array}$ & $w_{\mathrm{V}}=\frac{\left(\frac{b_{\mathrm{V}}}{2}\right)}{\sqrt{3}} \frac{100}{M_{\mathrm{N}, i}}$ & $w_{\mathrm{v}}=\frac{\left(\frac{b_{\mathrm{v}}}{2}\right)}{\sqrt{3}} \frac{100}{Y_{\mathrm{N}, i}}$ \\
\hline $\begin{array}{c}b_{\mathrm{L}} \\
\text { type B, } \\
\text { rectangular }\end{array}$ & $w_{\mathrm{L}}=\frac{\left(\frac{b_{\mathrm{L}}}{2}\right)}{\sqrt{3}} \frac{100}{M_{\mathrm{N}, i}}$ & $w_{\mathrm{L}}=\frac{\left(\frac{b_{\mathrm{L}}}{2}\right)}{\sqrt{3}} \frac{100}{Y_{\mathrm{N}, i}}$ \\
\hline
\end{tabular}

small enough to be neglected. From all these parameters, relative standard uncertainties are calculated according to Table 3.

Together with the standard uncertainty of the calibration torque $w_{\mathrm{M}, i}$, the standard uncertainties $w_{\mathrm{SV}, i}$ of the single measurement values can be determined from

$w_{\mathrm{SV}, i}=\sqrt{w_{\mathrm{M}, i}^{2}+2 \cdot w_{r}^{2}+w_{\mathrm{V}}^{2}+w_{\mathrm{L}}^{2}}$.

The standard uncertainties $w_{\mathrm{MV}, i}$ of the mean values calculated from (2) or (3) can be determined from

$w_{\mathrm{MV}, i}=\sqrt{w_{\mathrm{M}, i}^{2}+2 \cdot w_{r}^{2}+w_{b, i}^{2}+w_{\mathrm{V}}^{2}+w_{\mathrm{L}}^{2}}$.

In (10) and (11) it was taken into account that the measurement value is often the result of two readings, one at zero and one at the load point. It was assumed that both readings are affected by the resolution of the tool's setting or indication in the same manner. The resolution of the calibration device was not used in this formula directly since it had to be

Table 4. Sample calibration data for case A.

\begin{tabular}{ccccccc}
\hline \multirow{2}{*}{$\begin{array}{c}\boldsymbol{M}_{\mathrm{N}, \boldsymbol{i}} \\
\mathbf{N} \cdot \mathbf{m}\end{array}$} & & $\boldsymbol{j}=\mathbf{1}$ & $\mathbf{2}$ & $\mathbf{3}$ & $\mathbf{4}$ & $\mathbf{5}$ \\
\cline { 3 - 7 } & 20 & 20.2 & 20.1 & 20.3 & 20.3 & 20.1 \\
\hline $2=1$ & 60 & 60.4 & 60.5 & 60.6 & 60.4 & 60.6 \\
\hline 3 & 100 & 101.0 & 101.5 & 102.0 & 102.5 & 103.0 \\
\hline
\end{tabular}

Table 5. Calculated calibration results for case A.

\begin{tabular}{ccccccc}
\hline$M_{\mathrm{N}, \boldsymbol{i}}$ & \multicolumn{7}{c}{ in $\mathbf{N} \cdot \mathbf{m}$} \\
\cline { 2 - 7 } $\mathbf{N} \cdot \mathbf{m}$ & $\boldsymbol{Y}_{\boldsymbol{i}}$ & $\boldsymbol{f}_{\mathbf{q}, \boldsymbol{i}}$ & $\boldsymbol{b}_{\boldsymbol{i}}$ & $\boldsymbol{b}_{\mathbf{V}}$ & $\boldsymbol{b}_{\mathrm{L}}$ & $\boldsymbol{r}$ \\
\hline 20 & 20.2 & 0.20 & 0.20 & 0.20 & 0.10 & 0.10 \\
\hline 60 & 60.5 & 0.50 & 0.20 & 0.20 & 0.10 & 0.10 \\
\hline 100 & 102.0 & 2.00 & 2.00 & 0.20 & 0.10 & 0.10 \\
\hline
\end{tabular}

Table 6. Calculated uncertainties for case A.

\begin{tabular}{cccccccc}
\hline \multirow{2}{*}{$M_{\mathrm{N}, \boldsymbol{i}}$} & \multicolumn{7}{c}{ in \% } \\
\cline { 2 - 7 } $\mathrm{N} \cdot \mathrm{m}$ & $\boldsymbol{w}_{\mathrm{M}, \boldsymbol{i}}$ & $\boldsymbol{w}_{\mathrm{b}, \boldsymbol{i}}$ & $\boldsymbol{w}_{\mathrm{V}, \boldsymbol{i}}$ & $\boldsymbol{w}_{\mathrm{L}, \boldsymbol{i}}$ & $\boldsymbol{w}_{\boldsymbol{r}, \boldsymbol{i}}$ & $\boldsymbol{w}_{\mathrm{MV}, \boldsymbol{i}}$ & $\boldsymbol{W}_{\boldsymbol{i}}^{\prime}$ \\
\hline 20 & 0.05 & 0.289 & 0.289 & 0.144 & 0.144 & 0.481 & 1.963 \\
\hline 60 & 0.05 & 0.096 & 0.096 & 0.048 & 0.048 & 0.167 & 1.168 \\
\hline 100 & 0.05 & 0.577 & 0.058 & 0.029 & 0.029 & 0.585 & 3.169 \\
\hline
\end{tabular}


Table 7. Sample calibration data for case B.

\begin{tabular}{|c|c|c|c|c|c|c|}
\hline \multirow{2}{*}{\multicolumn{2}{|c|}{$\begin{array}{c}Y_{\mathrm{N}, i} \\
\mathrm{~N} \cdot \mathrm{m}\end{array}$}} & \multicolumn{5}{|c|}{$M_{\mathrm{A}, i, j}$ in $\mathrm{N} \cdot \mathrm{m}$} \\
\hline & & \multirow{2}{*}{$\begin{array}{r}\boldsymbol{j}=\mathbf{1} \\
19.8\end{array}$} & \multirow{2}{*}{$\frac{2}{19.9}$} & \multirow{2}{*}{$\frac{3}{19.7}$} & \multirow{2}{*}{$\begin{array}{c}4 \\
19.7\end{array}$} & \multirow{2}{*}{$\frac{\mathbf{5}}{19.9}$} \\
\hline$i=1$ & 20 & & & & & \\
\hline 2 & 60 & 59.6 & 59.5 & 59.4 & 59.6 & 59.4 \\
\hline 3 & 100 & 99.0 & 98.5 & 98.0 & 97.5 & 97.0 \\
\hline
\end{tabular}

Table 8. Calculated calibration results for case B.

\begin{tabular}{ccccccc}
\hline \multirow{2}{*}{$\begin{array}{r}\boldsymbol{Y}_{\mathrm{N}, \boldsymbol{i}} \\
\mathrm{N} \cdot \mathrm{m}\end{array}$} & $\boldsymbol{M}_{\boldsymbol{i}}$ & $\boldsymbol{f}_{\mathbf{q}, \boldsymbol{i}}$ & $\boldsymbol{b}_{\boldsymbol{i}}$ & $\boldsymbol{b}_{\mathrm{V}}$ & $\boldsymbol{b}_{\mathrm{L}}$ & $\boldsymbol{r}$ \\
\hline 20 & 19.8 & 0.20 & 0.20 & 0.20 & 0.10 & 0.10 \\
\hline 60 & 59.5 & 0.50 & 0.20 & 0.20 & 0.10 & 0.10 \\
\hline 100 & 98.0 & 2.00 & 2.00 & 0.20 & 0.10 & 0.10 \\
\hline
\end{tabular}

Table 9. Calculated uncertainties for case B.

\begin{tabular}{cccccccc}
\hline \multirow{2}{*}{$\boldsymbol{Y}_{\mathrm{N}, \boldsymbol{i}}$} & \multicolumn{7}{c}{ in \% } \\
\cline { 2 - 7 } $\mathrm{N} \cdot \mathrm{m}$ & $\boldsymbol{w}_{\mathrm{M}, \boldsymbol{i}}$ & $\boldsymbol{w}_{\mathrm{b}, \boldsymbol{i}}$ & $\boldsymbol{w}_{\mathrm{V}, \boldsymbol{i}}$ & $\boldsymbol{w}_{\mathrm{L}, \boldsymbol{i}}$ & $\boldsymbol{w}_{\boldsymbol{r}, \boldsymbol{i}}$ & $\boldsymbol{w}_{\mathrm{MV}, \boldsymbol{i}}$ & $\boldsymbol{W}_{\boldsymbol{i}}^{\prime}$ \\
\hline 20 & 0.05 & 0.289 & 0.289 & 0.144 & 0.144 & 0.481 & 1.963 \\
\hline 60 & 0.05 & 0.096 & 0.096 & 0.048 & 0.048 & 0.167 & 1.168 \\
\hline 100 & 0.05 & 0.577 & 0.058 & 0.029 & 0.029 & 0.585 & 3.169 \\
\hline
\end{tabular}

considered when the uncertainty of the calibration torques $w_{\mathrm{M}, i}$ was calculated.

As usual, the expanded relative uncertainties can be found by multiplying standard uncertainties with the corresponding coverage factor $k=2$. The expanded relative uncertainty of the single value $W_{\mathrm{SV}, i}$ should be the basis for a conformity evaluation as shown in Figure 2: the red line is the calibration torque, the dotted lines mark the permissible interval.

The uncertainty bar of the first value lies partly outside the permissible range and no conformity can be declared.

The relative deviation $A_{\mathrm{s}}$ defined in (1) now reads with the new designations introduced above in Table 2

- in case A as

$\frac{f_{\mathrm{q}}\left(M_{\mathrm{N}, i}\right)}{M_{\mathrm{N}, i}}=\frac{f_{\mathrm{q}, i}}{M_{\mathrm{N}, i}}=\frac{Y_{i}-M_{\mathrm{N}, i}}{M_{\mathrm{N}, i}}$, and

- in case B as
$\frac{f_{\mathrm{q}}\left(Y_{\mathrm{N}, i}\right)}{M_{i}}=\frac{f_{\mathrm{q}, i}}{M_{i}}=\frac{Y_{\mathrm{N}, i}-M_{i}}{M_{i}}$.

Due to the fact that this deviation is not a stochastic quantity but shows rather deterministic behaviour, it was not treated like an uncertainty. Therefore, it cannot be combined with the other uncertainties under the square root sign in (10) or (11). For the calibration results, uncertainty intervals $W_{i}^{\prime}$ will be calculated instead as the sum of the absolute value of the relative deviations and the expanded relative uncertainties.

The result for case (A) is

$W_{i}^{\prime}=\left|\frac{f_{\mathrm{q}}\left(M_{\mathrm{N}, i}\right)}{M_{\mathrm{N}, i}}\right| \cdot 100 \%+k \cdot w_{\mathrm{MV}, i}$,

and for case $\mathrm{B}$ it is

$W_{i}^{\prime}=\left|\frac{f_{\mathrm{q}}\left(Y_{\mathrm{N}, i}\right)}{M_{i}}\right| \cdot 100 \%+k \cdot w_{\mathrm{MV}, i}$.

Numerical examples

Case A - calibration controlled by the calibration facility's indication (not applicable to Type II tools):

Case B - calibration controlled by the torque tool:

The values and results given in Tables 4 to 9 can be taken as reference data sets for the validation of calculations.

\section{CONCLUSIONS}

The improvements of the ISO 6789 standard discussed here would allow this document to become a full calibration guide which is in accordance with the GUM. The methods proposed in this paper take into account the most important and relevant influencing quantities which are known to date.

\section{REFERENCES}

[1] ISO 6789:2003, Assembly tools for screws and nuts - Hand torque tools - Requirements and test methods for design conformance testing, quality conformance testing and recalibration procedure.

[2] Evaluation of measurement data - Guide to the expression of uncertainty in measurement, Internet: http://www.bipm.org/

[3] L. G. Bochese, "Uncertainty of Measurement in Torque Wrench Calibration", 2003 NCSL International Workshop and Symposium, Tampa, Florida, USA, August 17-21, 2003.

[4] Source: "http://de.wikipedia.org/w/index.php?title=Datei: Drehmomentschl\%C3\%BCssel_3360.jpg\&filetimestamp $=201202$ 25121256", Author: Torquemaster.

[5] ISO/IEC 17025:2005, General requirements for the competence of testing and calibration laboratories. 